\title{
The orbital stability of a test body motion in the field of two mas- sive bodies
}

\author{
Medeu Abishev ${ }^{1,2,3, \star}$, Saken Toktarbay ${ }^{1,2, \star \star}$ Aigerim Abylayeva $^{1,2,}$, and Amanhan Talkhat ${ }^{1,2,}$ \\ ${ }^{1}$ IETP, \\ ${ }^{2}$ Physical and Technical Faculty, Al-Farabi Kazakh National University, al'-Farabi avenue, 71, Almaty 050040, \\ Kazakhstan \\ ${ }^{3}$ Institute of Gravitation and Cosmology, \\ RUDN University, \\ Miklukho-Maklaya St.,6, Moscow 117198, Russia
}

\begin{abstract}
We investigate the orbital stability of a test particle motion in the restricted three-body problem where all bodies have their own rotation. We have shown that it is possible to get some insight into the stability properties of the motion of test particles in restricted three-body problem, without knowing the exact solutions of the relativistic motion equations.
\end{abstract}

\section{Introduction}

In the works dedicated to the relativistic three-body problem, some peculiarities of private problems were considered [1-3]. But in some cases, as we believe, cosmogonic general relativistic effects can be substantial, even determining the evolution of a system of bodies, especially when it comes to stable orbital motions of celestial bodies. Therefore, the development of relativistic planetary cosmogony and the study of the evolution of planetary systems in the framework of General relativity (GR) is very important. The orbital stability of two rotating bodies investigated in $[4,5]$. As well as, the orbital stability problem of circular motion of a test body in the restricted three-body problem with the relativistic corrections investigated in [6], where the appropriate conditions are

$$
U_{1}<<c^{2}, \quad U_{2}<<U_{1}, \quad U_{1} / U_{2} \approx v^{2} / c^{2},
$$

where $U_{1}, U_{2}$ are the potentials of central and second bodies in the considered region respectively. In that case, all the bodies do not have their own rotation. The resting position of the central body (the first body) coincides with the reference point of coordinates while the second body moves along the circle around the central body and is not subjected to the disturbance. The test body moves in a perturbed circular orbit. This problem belongs to the class of quasi-keplerian, Therefore, in this case, we can investigate the orbital stability problem by the adiabatic theory of motion in GR mechanics which was developed by academician M.M. Abdildin [4].

\footnotetext{
$\star$ e-mail: abishevme@gmail.com

$\star \star$ e-mail: saken.yan@yandex.com
} 


\section{The equation of motion of a test body}

According to the adiabatic theory, the evolutionary motion of the test (third) body describes the average change of its orbital momentum. Therefore, we write down the orbital angular momentum of the test body

$$
\vec{M}=\left[\vec{r}_{3}, \vec{p}_{3}\right]
$$

and the time derivative from it

$$
\dot{\vec{M}}=\left[\dot{\vec{r}}_{3}, \vec{p}_{3}\right]+\left[\vec{r}_{3}, \dot{\vec{p}}_{3}\right]
$$

where

$$
\dot{\vec{r}}_{3}=\frac{\partial H}{\partial \vec{p}_{3}}, \quad \dot{\vec{p}}_{3}=-\frac{\partial H}{\partial \vec{r}_{3}},
$$

are found by means of the equations of Hamilton from the Hamilton function of the system.

In [6], Abishev et al., the relativistic equation of translational motion of a test body in the field of two bodies in the mechanics of general relativity is investigated by the asymptotic methods of the adiabatic theory through the process of averaging the corresponding equations using the vector elements $\vec{M}$ (the orbital moment) and $\vec{A}$ (the Laplace vector). It is shown that in this case the orbit of the test body is stable in the form of circular orbit.

In the present work, we consider the orbital stability problem for the circular motion of a test body in a restricted three-body problem when all bodies have their own rotation. We derived the Lagrange function of a system of bodies up to the second-order terms. It is quite sufficient for the problems of relativistic celestial mechanics that the influence of the internal structure of bodies can generally be neglected and omitted from all terms associated with taking this influence into account. We confine ourselves to the zeroth order terms of the expansion in powers of the ratio of body size to their mutual distances.

This work is organized as follows. In Sec. 2, we present the explicit form of the Lagrangian of the three body, containing an additional rotating term. We also present an equation of motion for the test body. We use the adiabatic theory of motion to analyse the orbital stability of test body. In Sec.3 we will present the averaging equation of motion. Finally, in Sec. 4, we discuss our results.

The Lagrangian for the translational and rotational motion of the three rotating bodies without interior structure can be represented in this form:

$$
L=L^{(0)}+L^{(*)} .
$$

Consequently, the Hamiltonian function can be represented in this form:

$$
H=H^{(0)}+H^{(*)}
$$

According to the equations (3) and (4) the time derivative of orbital angular momentum and equation of motion can be represented in the following form:

$$
\dot{\vec{M}}=\dot{\vec{M}}^{(0)}+\dot{\vec{M}}^{(*)}
$$




$$
\dot{\vec{r}}_{i}=\frac{\partial H^{0}}{\partial \vec{p}_{i}}+\frac{\partial H^{*}}{\partial \vec{p}_{i}}=\dot{\vec{r}}_{i}^{(0)}+\dot{\vec{r}}_{i}^{(*)}
$$

and

$$
\dot{\vec{p}}_{i}=\frac{\partial H^{(0)}}{\partial \vec{r}_{i}}+\frac{\partial H^{(*)}}{\partial \vec{r}_{i}}=\dot{\vec{p}}_{i}^{(0)}+\dot{\vec{p}}_{i}^{(*)}
$$

where (0) term is for the three point like masses and it's explicit form was presented in the work [6] by Abishev at al., and (*) is the additional term which related to the rotation of the body.

From the general form of the Lagrangian for $\mathrm{N}$ bodies $[5,7,8]$, we can derive the rotational term of Lagrangian as following:

$$
\begin{aligned}
& L^{(*)}=\sum_{i=1}^{3} \omega_{i}^{2} I_{i}\left(1+\frac{1}{c^{2}}\right)-\frac{1}{2 c^{2}}\left[I_{2}\left(\overrightarrow{\omega_{2}} \dot{\overrightarrow{r_{2}}}\right)^{2}+I_{3}\left(\overrightarrow{\omega_{3}} \dot{\vec{r}}_{3}\right)^{2}\right] \\
& -\frac{\gamma}{c^{2}}\left\{\frac{\overrightarrow{r_{2}} \cdot \dot{\overrightarrow{r_{2}}}}{\left|\overrightarrow{r_{2}}\right|^{3}}\left[3 m_{1} I_{2} \omega_{2}+4 m_{2} I_{1} \omega_{1}\right]-\frac{\overrightarrow{r_{3}} \cdot \dot{\overrightarrow{r_{3}}}}{\left|\overrightarrow{r_{3}}\right|^{3}}\left[3 m_{1} I_{3} \omega_{3}+4 m_{3} I_{1} \omega_{1}\right]\right. \\
& \left.+\frac{\vec{r}_{2}-\vec{r}_{3}}{\left|\overrightarrow{r_{2}}-\overrightarrow{r_{3}}\right|^{3}}\left[\left(3 \dot{\vec{r}}_{3}-4 \dot{\overrightarrow{r_{2}}}\right) m_{2} I_{3} \omega_{3}-\left(3 \dot{\vec{r}}_{2}-4 \dot{\vec{r}}_{3}\right) m_{3} I_{2} \omega_{2}\right]\right\} \\
& -\frac{\gamma}{2 c^{2}} \frac{m_{2} I_{2}+m_{3} I_{3}}{\left|\vec{r}_{2}-\vec{r}_{3}\right|^{3}}\left[\dot{\vec{r}}_{2} \cdot \dot{\vec{r}}_{3}-\frac{3\left(\left(\vec{r}_{2}-\vec{r}_{3}\right) \cdot \dot{\vec{r}}_{2}\right)\left(\left(\vec{r}_{2}-\vec{r}_{3}\right) \cdot \dot{\vec{r}}_{3}\right)}{\left|\vec{r}_{2}-\vec{r}_{3}\right|^{2}}\right] \\
& -\frac{3 \gamma}{c^{2}}\left[\frac{1}{\left|\vec{r}_{2}\right|}\left(m_{2} \omega_{2}^{2} I_{2}+m_{2} \omega_{1}^{2} I_{1}\right)+\frac{1}{\left|\vec{r}_{3}\right|}\left(m_{1} \omega_{3}^{2} I_{3}+m_{3} \omega_{1}^{2} I_{1}\right)+\frac{1}{\left|\vec{r}_{2}-\vec{r}_{3}\right|}\right. \\
& \left.\times\left(m_{2} \omega_{3}^{2} I_{3}+m_{3} \omega_{2}^{2} I_{2}\right)\right]+\frac{\gamma^{2}}{2 c^{2}}\left\{\frac{m_{1} I_{2}+m_{2} I_{1}}{\left|\vec{r}_{2}\right|^{2}}+\frac{m_{1} I_{3}+m_{3} I_{1}}{\left|\vec{r}_{3}\right|^{2}}\right. \\
& +\frac{m_{2} I_{3}+m_{3} I_{2}}{\left|\vec{r}_{2}-\vec{r}_{3}\right|^{2}}+m_{1} m_{2} I_{3} \frac{\vec{r}_{3}\left(\vec{r}_{3}-\vec{r}_{2}\right)}{\left|\vec{r}_{3}\right|^{3}\left|\vec{r}_{2}-\vec{r}_{3}\right|^{3}}+m_{2} m_{2} I_{1} \frac{\vec{r}_{2} \cdot \vec{r}_{3}}{\left|\vec{r}_{3}\right|^{3}\left|\vec{r}_{2}-\vec{r}_{3}\right|^{3}} \\
& \left.+m_{1} m_{3} I_{2} \frac{\left(\vec{r}_{2}-\vec{r}_{3}\right) \cdot \vec{r}_{2}}{\left|\vec{r}_{2}-\vec{r}_{3}\right|^{3}\left|\vec{r}_{2}\right|^{3}}\right\}-\frac{\gamma}{c^{2}}\left\{4\left[\frac{I_{1} I_{2}}{\left|\vec{r}_{2}\right|^{3}}+\frac{I_{1} I_{3}}{\left|\vec{r}_{3}\right|^{3}}+\frac{I_{2} I_{3}}{\left|\vec{r}_{2}-\vec{r}_{3}\right|^{3}}\right]\right. \\
& -3\left[\frac{\left(\vec{r}_{2} \vec{\omega}_{1}\right) \cdot\left(\vec{r}_{2} \vec{\omega}_{2}\right)}{\left|\vec{r}_{2}\right|^{3}}+\frac{\left(\vec{r}_{3} \vec{\omega}_{1}\right)\left(\vec{r}_{3} \vec{\omega}_{3}\right)}{\left|\vec{r}_{3}\right|^{3}}+\frac{\left(\left(\vec{r}_{2}-\vec{r}_{3}\right) \vec{\omega}_{2}\right)\left(\left(\vec{r}_{2}-\vec{r}_{3}\right) \vec{\omega}_{3}\right)}{\left|\vec{r}_{2}-\vec{r}_{3}\right|^{3}}\right] .
\end{aligned}
$$

We can find the Hamiltonian using the following well known relation:

$$
H=\vec{v}_{i} \frac{\partial L}{\partial \vec{v}_{i}}-L
$$

and by taking into account the Eqs. (5) and (6) we can write down the additional term of the Hamiltonian and the derivatives of the canonical variables of the test body.

\section{Averaging equation of motion}

According to the adiabatic theory, in order to obtain the evolutionary equations of motion one needs to integrate the equation (7) for the repetition period of system configurations $\mathrm{T}$ (synodic period of the test body):

$$
\overline{\dot{\vec{M}}}=\frac{1}{T} \int_{0}^{T} \dot{\vec{M}} d t
$$


where

$$
T=\frac{2 \pi}{\omega_{2}-\omega_{3}} .
$$

In fact, the preliminary results show that it is possible to find the time derivative of the angular momentum, and then integrate the equation to find the average change in the orbital angular momentum. In this case, it can be shown that under certain conditions which satisfy the stability conditions for the orbital motion of the test body we can find solutions for the orbit of test body in the restricted three body problem.

\section{Conclusions}

In this work, we considered the orbital stability problem for the circular motion of a test body in the restricted three-body problem within the framework of GR, when all bodies have their own rotation. We derived equation of motion from the Lagrange function with the rotational terms. The orbital stability of the test body is investigated by the adiabatic theory of motion in the GR. Moreover, we derived the explicit form of the corresponding motion equation and the time derivative of momentum. The resulting equations was integrated (averaged) by the sinodic period of the test body, which eliminates perturbations, leaving only evolutionary effects. The result of the integral is confirmed that the circular motion will be stable if the spins of all bodies are collinear within a plane. The quasicircular orbits of the test body will be analyzed in a forthcoming works.

\section{Acknowledgments}

This work has been partially supported by the Ministry of Education and Science (Kazakhstan), in particular by grant to project "Restricted three-body problem in the General Relativity"

\section{References}

[1] Maindl T. I.\& Dvorak R. Astron.Astrophys. 290, 335 (1994)

[2] Imai T1, Chiba T, Asada H. Phys. Lett. B 98, (2007).

[3] Kei Yamada, Hideki Asada . Phys. Rev. D 82,104019 (2010).

[4] Abdil'din M. M., Abishev M. E., Beissen N. A. Gravitation and Cosmology V15, 141 (2009).

[5] Brumberg A.A. Relativistic celestial mechanics ( Nauka, 1972), 382 p.

[6] Abishev M. E., Toktarbay, S., Zhami B. A. Gravitation and Cosmology. V20, 149 (2014).

[7] Landau L.D., Lifshitz A.I.The Classical Theory of Fields ( Nauka, 1973), Vol. 2. 402 p.

[8] Abdildin M.M. Mechanins of Einstein theory of gravity( Alma-Ata: Nauka, 1988), 198 p. 